\title{
JSEG Based Color Separation of Tongue Image in Traditional Chinese Medicine
}

\author{
Yonggang Wang ${ }^{1}$, Yue Zhou ${ }^{1}$, Jie Yang ${ }^{1}$, and Yiqin Wang ${ }^{2}$ \\ 1 Institute of Image Processing \& Pattern Recognition, \\ Shanghai Jiaotong University, Shanghai, China, 200030 \\ \{yonggangwang, zhouyue, jieyang\}@sjtu.edu.cn \\ 2 School of Basic Medicine, Shanghai University of TCM, Shanghai, China, 200032 \\ wangyq@stcm.edu.cn
}

\begin{abstract}
The process of color separation of tongue image in traditional Chinese medicine (TCM) is decomposed into two steps: region partition and color classification. In the first step, a partition method based on JSEG is proposed to obtain homogenous regions in the tongue. In the second step, a priori template and some standard color patches are designed to assist the classification according to a priori knowledge from the TCM experts, where the nearest neighbour classifier is applied for color classification. The experimental results show that the proposed approach is greatly promising to computerized tongue diagnosis.
\end{abstract}

\section{Introduction}

Traditional Chinese medicine (TCM) consists of a systematized methodology of medical treatment, where much special physiological information could be obtained through analyzing the patient's tongue. The experienced TCM doctors usually incline to use the information of the patient's tongue to determine his disease and health conditions. Unfortunately, the traditional tongue diagnosis has been impeded because this diagnostic process involves a great deal of subjective information and experiential knowledge. Recently, more and more attention has been focused on building objective standards for tongue diagnosis and as a result, several tongue diagnosis systems based on image analysis theory [1-4] have been developed for the purpose of the diagnosis and treatment of patients. These systems include a series of algorithms for tongue image processing, e.g. color calibration, tongue extraction, color separation of tongue image, etc. The purpose of color calibration is to keep the consistency and repeatability of colors transmitted from image acquiring device (e.g. digital camera) to monitor so as to facilitate doctor's diagnosis and further image processing. Some standard color patches are usually used to build the calibration model. Before analyzing the tongue features, tongue extraction process is needed to cut out the tongue body from its surroundings. Here we mainly discuss the color separation algorithm of substance and coat which cover on the surface of tongue. It is very important to carry out the quantitative analysis of the tongue image. 
However, some drawbacks exist in these algorithms as follows. First, these algorithms carry out pixel-wise color recognition in the tongue image, which abandons the spatial correlations between pixels and neglects the local properties in the tongue image. Second, they are not fully associated with a priori knowledge of TCM. Third, such supervised classifiers as SVM and supervised FCM [3,4] are adopted to classify the colors of substance and coat, and thereby much tedious work like sample collecting and learning need be implemented.

The impetus of this research is to suggest a new algorithm to overcome the problems mentioned above. We discompose the process of color separation of the tongue image into two steps, i.e. region partition and color classification. In the first step, JSEG, a newly proposed method for unsupervised segmentation, is used to partition the tongue into several homogenous regions. Next in the second step, a priori template induced by the TCM experts' experience, is designed and applied to detect tongue substance and coat. A nearest neighbour classifier is used to color classification. Note that the color patches which have been used in color calibration and designed according to a priori knowledge, are used as the reference samples in the nearest neighbour classifier. Fig. 1 show a part of these color patches.

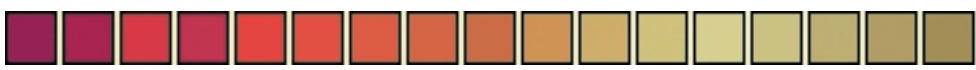

Fig. 1. Some color patches

This paper is organized as follows. Section 2 depicts the JSEG based region partition method. Section 3 discusses the color classification using a priori template and much expert's knowledge. Section 4 tests the proposed algorithm and section 5 concludes the paper.

\section{JSEG Based Region Partition}

\subsection{Local Properties of Tongue Image}

In the tongue image, the colors of the tongue substance are classified as light pink, rose pink, red and purple. And the coat colors are classified as white, yellow, brown and black. In general, the light pink substance and flimsily white coat are considered to be healthy. It can be observed that the tongue image is a typical kind of color-texture image and has locally different color distribution. Moreover, according to TCM principles, the conclusions drawn by doctors are a kind of region-based description of color distribution of tongue substance and coat. Following this logic, we firstly divide the tongue image into various homogenous regions with similar colors and textures. On the basis of this partition, we represent the colors in every region quantitatively so that some important features, e.g. substance colors, coat colors as well as their distribution, could be obtained. 


\subsection{JSEG Algorithm}

JSEG is a new method for unsupervised segmentation of color-texture regions in images which is presented in [5]. The basic idea of the JSEG method is to separate the segmentation process into two stages: color quantization and spatial segmentation. In the first stage, colors in the image are quantized to several representative classes that can be used to differentiate regions in the image. This quantization is performed in the color space without considering the spatial distributions of the colors. Then, the image pixel values are replaced by their corresponding color class labels, thus forming as a special kind of texture composition. In the second stage, a criterion for "good" segmentation is applied to local windows in the class-map. This results in the "J-image", in which high and low values correspond to possible boundaries and interiors of color-texture regions. A region growing method is then used to segment the image based on the multiscale J-images. The decoupling of color similarity from spatial distribution in the JSEG allows for the development of more tractable algorithms for each of the two processing stages. In fact, one of the good color quantization algorithms, called PGF [6], has been used in the original JSEG method. The JSEG algorithm is tested to show very robust to a variety of images.

In our research, the JSEG algorithm is used to partition different regions on the surface of the tongue image. Fig. 2 shows a partition example. Obviously in the result image, the tongue body is segmented into four regions, where region $\mathrm{C}$ corresponds to its substance while regions A, B and D correspond to its coat.

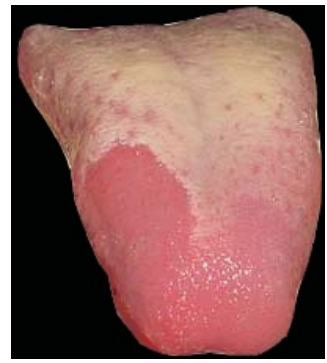

(a)

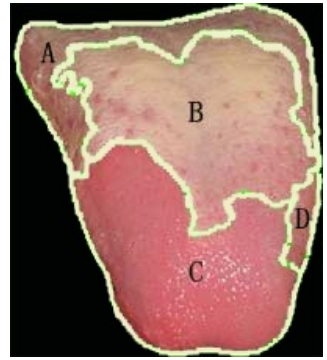

(b)

Fig. 2. An example of JSEG based region partition: (a) a tongue image and (b) its partition result

\section{Color Classification}

The distribution of substance and coat on the surface of tongue has properties of complexness and diversity, although, some principles could be derived. Most of the previous work didn't consider these principles. Hence, their classification accuracy was not too high. In our research, we have applied a priori knowledge as much as possible to improve the accuracy. 


\subsection{Priori Template}

Under the guide of the TCM experts, together with a great deal of observation, we summarize several principles about the distribution of substance and coat in tongue as follows. i) There usually exists much coat at the root of tongue. ii) There usually exists much substance at the tip of tongue. iii) As regard to the two sides of tongue, the probability of substance is larger than that of coat. iv) There is a transition of dark to light, i.e. thick to thin coat from the root to the tip of tongue. v) In a tongue body, generally, the amount of coat is more than substance.

According to these principles, we design a template to assist the following color separation. This template, shown in Fig. 3, divides a tongue into six parts whose sizes are labeled in detail. Part I is tongue root region, part II is middle-

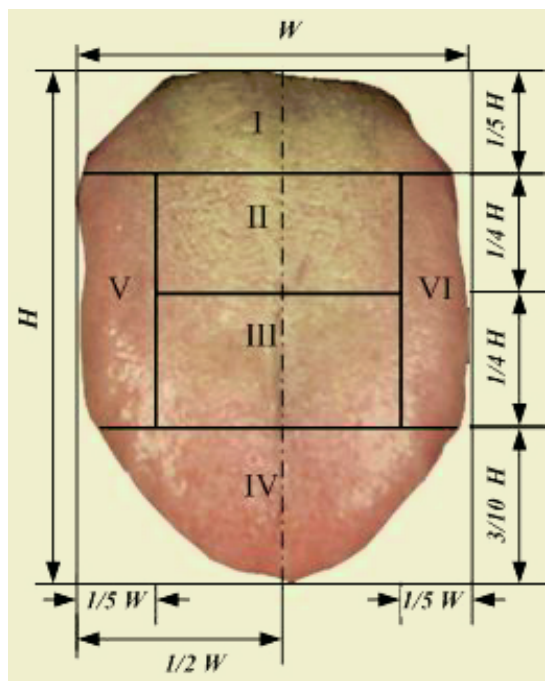

Fig. 3. Priori template

root region, part III is middle-tip region, part IV is tip region and parts V and VI are tongue side regions respectively. Besides, we plan out the following priority strategies for each sub-region $R_{i}$ obtained from the JSEG based partition.

- If $R_{i}$ falls into part I, it will be classified into the coat class as long as $10 \%$ of its pixels have coat colors.

- If $R_{i}$ falls into part II, it will be classified into the coat class as long as $40 \%$ of its pixels have coat colors.

- If $R_{i}$ falls into part III, it will be classified into the substance class as long as $40 \%$ of its pixels have substance colors.

- If $R_{i}$ falls into part IV, it will be classified into the substance class as long as $20 \%$ of its pixels have substance colors.

- If $R_{i}$ falls into part $\mathrm{V}$ or VI, it will be classified into the substance class as long as $30 \%$ of its pixels have substance colors. 
Note that $R_{i}$ will be considered to fall into a certain part only if more than $75 \%$ of its pixels fall into this part.

\subsection{Substance and Coat Color Classification}

To determine the color label of a pixel in the tongue image, a nearest neighbour classifier is used. The classifier is widely used in pattern recognition tasks (see Chapter 4 in [7]). As mentioned in the beginning, we use the color patches in Fig. 1 as the reference samples. These color patches are designed in the color calibration stage by the experienced TCM experts and contain the usual colors of substance and coat in TCM tongue diagnosis. Since in the JSEG method the CIE LUV color space is adopted, here we evaluate the distances of pixels' features in this space. The color space has a perceptually uniform property where the image components are reasonably uncorrelated with each other. Euclidean distance in this space is used to classify any pixel in each homogeneous subregion into its nearest neighbour color class corresponding to the least distance. Then the priority strategies above are applied and the class of the sub-region belonging to substance or coat is determined.

\section{Experimental Results}

The proposed algorithm is tested in our computerized tongue diagnosis system. This system provides a stable and consistent sampling environment which includes several main components as follows: standard light source with $5000 \mathrm{~K}$ color temperature, Canon-G5 CCD digital camera with a resolution of $1024 \times 768$ pixels, Dell Dimension8200 workstation, dark chest with face supporting structure as well as tongue image analysis software. Using this system, we have collected thousands of tongue images from clinical patients. The colors occurring in different zones of each tongue image are judged simultaneity by more than three experienced TCM doctors. To avoid a large variation among different doctors for the same tongue image, we pick out over 600 samples with consistent evaluation on colors by those doctors and test the proposed algorithm on them. Over $90 \%$ of the color separation results are coincident with the doctors' conclusions.

Fig. 4 gives two examples by our method. We adopt pseudocolors to represent the different color classes of substance and coat after color separation, e.g. light pink corresponding to light pink substance, grey corresponding to white coat, yellow corresponding to yellow coat and black corresponding to black coat.

\section{Conclusions}

Tongue color separation is an important stage in tongue diagnosis systems based on image analysis. In this paper, a new method is proposed, which fully considers the local property of the tongue image as well as a priori knowledge. The basic idea of this method is to decompose the color separation problem into two steps, 


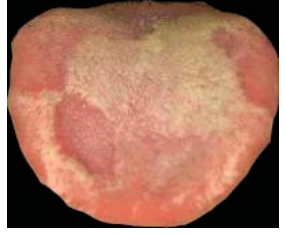

(a)

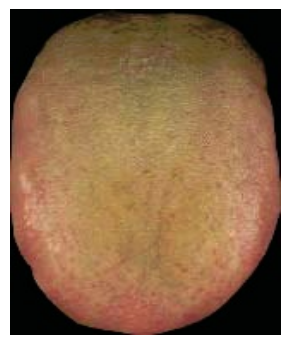

(d)

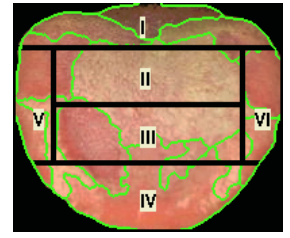

(b)

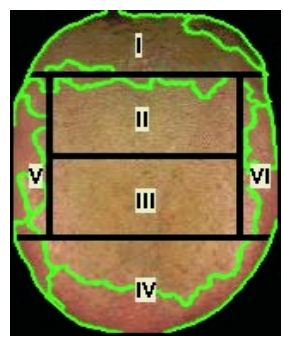

(e)

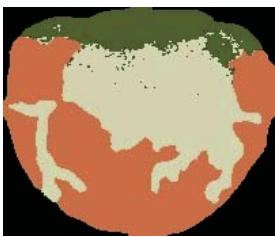

(c)

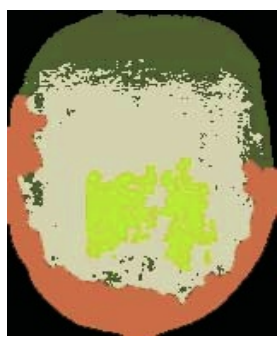

(f)

Fig. 4. Examples of color separation. (a) and (d) are the original tongue images; (b) and (e) are JSEG based partition results superimposed with the priori template; (c) and (f) are color separation results in a pseudocolors representation

region partition and color classification. In the first step, the tongue image is divided into various homogenous sub-regions by the aid of the JSEG algorithm. In the second step, a nearest neighbour classifier is used to further determine these sub-regions to belong to the substance or coat class. Finally, the approach proposed here is tested to show much potential to computerized tongue diagnosis.

\section{References}

1. Jang, J.H., Kim, J.E., Park, K.M., et al: Development of the digital tongue inspection system with image analysis. In: IEEE Proc. of the 2nd Joint EMBS/BMES Conf. Volume I., Houston, TX, USA (2002) 1033-1034

2. Chiu, C.C.: A novel approach based on computerized image analysis for traditional chinese medical diagnosis of the tongue. Computer Methods and Programs in Biomedicine 61 (2000) 77-89

3. Zhao, Z.X., Wang, A.M., Shen, L.S.: An automatic tongue analyzer of chinese medicine based on color image processing. In: The 4th Int. Conf. on Electronic Measurement and Instruments Conf. Proc., Harbin, China (1999) 830-834

4. Wang, Y.G.: Research on pragmatizing the tongue image analysis instrument of traditional chinese medicine. Master's thesis, Beijing Polytechnic University (2001)

5. Deng, Y.N., Manjunath, B.S.: Unsupervised segmentation of color-texture regions in images and video. IEEE Trans. on PAMI 23 (2001) 800-810

6. Deng, Y.N., Kenney, C., Moore, M.S., et al: Peer group filtering and perceptral color image quantization. In: Proc. IEEE Int. Symp. Circuits and Systems. Volume 4., Orlando, FL (1999) 21-24

7. Duda, R.O., Hart, P.E., Stork, D.G.: Pattern classification. Second edn. John Wiley \& Sons, New York (2001) 